\title{
TUMORES EN COSTA RICA: ÉNFASIS EN CÁNCER COLORRECTAL
}

\section{Young Leitón, Jean Carlo}

Médico General, Instituto Nacional de Seguros, La Uruca, San José, Costa Rica

Resumen: Actualmente en Costa Rica el cáncer colorrectal tiene una incidencia en hombres de 9,49\% por cada 100000 habitantes, convirtiéndolo en el cuarto tumor más frecuente en el país, mientras que en las mujeres ocupa el sexto lugar en incidencia con un 9,22\%. Además, en mortalidad es el quinto tumor más letal en hombres y en mujeres se convirtió en la tercera causa de muerte más frecuente. Este último punto es el más significativo, ya que con respecto a la mortalidad en mujeres pasa de ser la cuarta causa en el año 2000 a ser la tercera en el 2010, por encima del cáncer de cérvix. Los datos anteriores se basan en estudios del Ministerio de Salud para el año 2007 (incidencia) y 2010 (mortalidad).

Se han descrito múltiples factores de riesgo, entre los cuales se encuentran aquellos modificables como la dieta, el fumado y la obesidad y los no modificables como los síndromes polipósicos y el cáncer de colon hereditario no asociado a estos.

Usualmente los síntomas tempranos del cáncer de colon y recto son inespecíficos, por lo que se muestran hasta en estadios más avanzados de la enfermedad cuando la clínica es más evidente, siendo el sangrado rectal uno de los síntomas más frecuentes.

El tratamiento dependerá de si el tumor se encuentra en etapas tempranas o avanzadas, en donde la intervención quirúrgica adecuada, junto con quimioterapia adyuvante ha demostrado mejores índices en la curación.

Palabras clave: cáncer colon, colonoscopia, cáncer rectal 


\title{
TUMORS IN COSTA RICA; EMPHASIS ON COLORECTAL CANCER
}

\begin{abstract}
Currently in Costa Rica colorectal cancer has an incidence of 9,49\% in men for every 100000 inhabitants, making it the fourth most common cancer in the country, while women ranked sixth in incidence with a 9,22 \%. Furthermore, mortality is the fifth most deadly tumor in men and women became the third most common cause of death. This last point is the most significant, because with respect to mortality in women happens to be the fourth leading cause in 2000 to be the third in 2010, up from cervical cancer. The above data are based on studies by the Ministry of Health for the year 2007 (incidence) and 2010 (mortality). Described multiple risk factors, among which are those modifiable as diet, smoking and obesity and nonmodifiable as polyposis syndromes and hereditary colon cancer is not associated with these. Usually early symptoms of colorectal cancer are nonspecific, so they show up in more advanced stages of the disease when the clinic is more evident, rectal bleeding being one of the most common symptoms. Treatment will depend on whether the tumor is in early or advanced stages, where appropriate surgery along with adjuvant chemotherapy has shown better in healing rates.
\end{abstract}

Key words: colon cancer, colonoscopy, rectal cancer

\section{INTRODUCCIÓN}

En Costa Rica, al igual que en el ámbito mundial, el cáncer de colon es una de las tumoraciones más letales junto con los de próstata, mama, pulmones y estómago.

Por décadas se ha asociado el cáncer colorrectal con países altamente industrializados. En Norte América la incidencia en hombres es del $9 \%$ de todos los casos de las tumoraciones nuevas, mientras que la incidencia femenina es del $10 \%$ [1]. Se estima que aproximadamente 150000 norteamericanos anualmente son diagnosticados con cáncer colorrectal, de los cuales un tercio de los pacientes no va a poder superar la enfermedad [2].
Sin embargo, en Costa Rica el cáncer colorrectal también muestra una incidencia en hombres de 9,49\% por cada 100000 habitantes (Fig.1), convirtiéndolo en el cuarto tumor más frecuente en este género, mientras que en mujeres ocupa el quinto lugar en incidencia con un 9,22\% (Fig. 2) [3]. Tales datos demuestran una misma tendencia que en los países industrializados y se rompe el paradigma de que es más común en estos que en países como el nuestro. Esto basado en los datos del Ministerio de Salud con la actualización para el año 2007 [3].

Inclusive en cuanto a su mortalidad, en Costa Rica, para la población masculina, ocupa el quinto lugar (Fig. 3), solo superado por el cáncer de hígado, el de pulmón, el de estómago y el de próstata, que ocupan el cuarto, tercero, segundo y primer lugar, respectivamente. Por su parte, en el caso de las mujeres, 
Fig. 1: Incidencia de tumores malignos más frecuentes en hombres, años 2000-2007. Tasas ajustadas por 100000 hombres.

\begin{tabular}{|c|c|c|c|c|c|c|c|c|}
\hline \multirow[t]{2}{*}{$\begin{array}{r}63 \\
58 \\
53 \\
48 \\
43 \\
38 \\
33 \\
28 \\
23 \\
18 \\
13 \\
8 \\
3\end{array}$} & \multicolumn{4}{|c|}{$y$} & \multicolumn{4}{|c|}{ 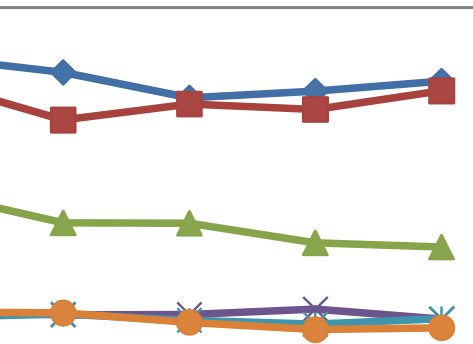 } \\
\hline & 2000 & 2001 & 2002 & 2003 & 2004 & 2005 & 2006 & 2007 \\
\hline -próstata & 46,01 & 48,76 & 57,09 & 58,43 & 55,71 & 50,95 & 52,15 & 54,03 \\
\hline - p piel & 42,39 & 51,52 & 48,74 & 53,61 & 46,74 & 49,75 & 48,72 & 52,22 \\
\hline —-estómago & 34,83 & 34,34 & 28,89 & 32,88 & 27,48 & 27,4 & 23,72 & 22,96 \\
\hline ×pulmón & 11,43 & 11,82 & 10,23 & 11,01 & 10,21 & 10,26 & 11,31 & 9,39 \\
\hline$*$ colon & 6,49 & 10,32 & 9,07 & 9,81 & 10,33 & 9,21 & 8,45 & 9,49 \\
\hline -shr** & 8,31 & 9,8 & 9,65 & 10,75 & 10,57 & 8,79 & 7,45 & 7,79 \\
\hline
\end{tabular}

**SHR: Sistema hematopoyético y retículo endotelial

Fuente: Ministerio de Salud, Dirección Vigilancia de la Salud, Registro Nacional Tumores, Costa Rica

Fig. 2: Incidencia de tumores malignos más frecuentes en mujeres años 2000-2007.

Tasas ajustadas por 100000 mujeres.

\begin{tabular}{|c|c|c|c|c|c|c|c|c|}
\hline \multirow[t]{2}{*}{$\begin{array}{r}53 \\
48 \\
43 \\
38 \\
33 \\
28 \\
23 \\
18 \\
13 \\
8 \\
3\end{array}$} & \multicolumn{8}{|c|}{$x$} \\
\hline & 2000 & 2001 & 2002 & 2003 & 2004 & 2005 & 2006 & 2007 \\
\hline —próstata & 35,99 & 48,3 & 43,88 & 45,68 & 40,41 & 44,11 & 43,69 & 48,19 \\
\hline$\neg$ piel & 40,19 & 40,35 & 40,27 & 41,45 & 43,7 & 41,13 & 41,78 & 42,71 \\
\hline estómago & 50,5 & 46,94 & 45,57 & 48,64 & 42,39 & 42,72 & 35,11 & 30,65 \\
\hline * pulmón & 19,54 & 16,01 & 16,24 & 17,78 & 16,54 & 14,2 & 15,81 & 13,72 \\
\hline$*$ colon & 9,09 & 8,93 & 9,93 & 10,83 & 10,94 & 12,62 & 14,18 & 13,09 \\
\hline - shr** & 7,69 & 9,09 & 7,47 & 9,34 & 10,88 & 9,84 & 8,45 & 9,22 \\
\hline
\end{tabular}

Fuente: Ministerio de Salud, Dirección Vigilancia de Salud, Registro Nacional Tumores, Costa Rica 
Fig. 3: Mortalidad de tumores malignos más frecuentes en hombres, años 2000-2010. Tasas ajustadas por 100000 hombres.

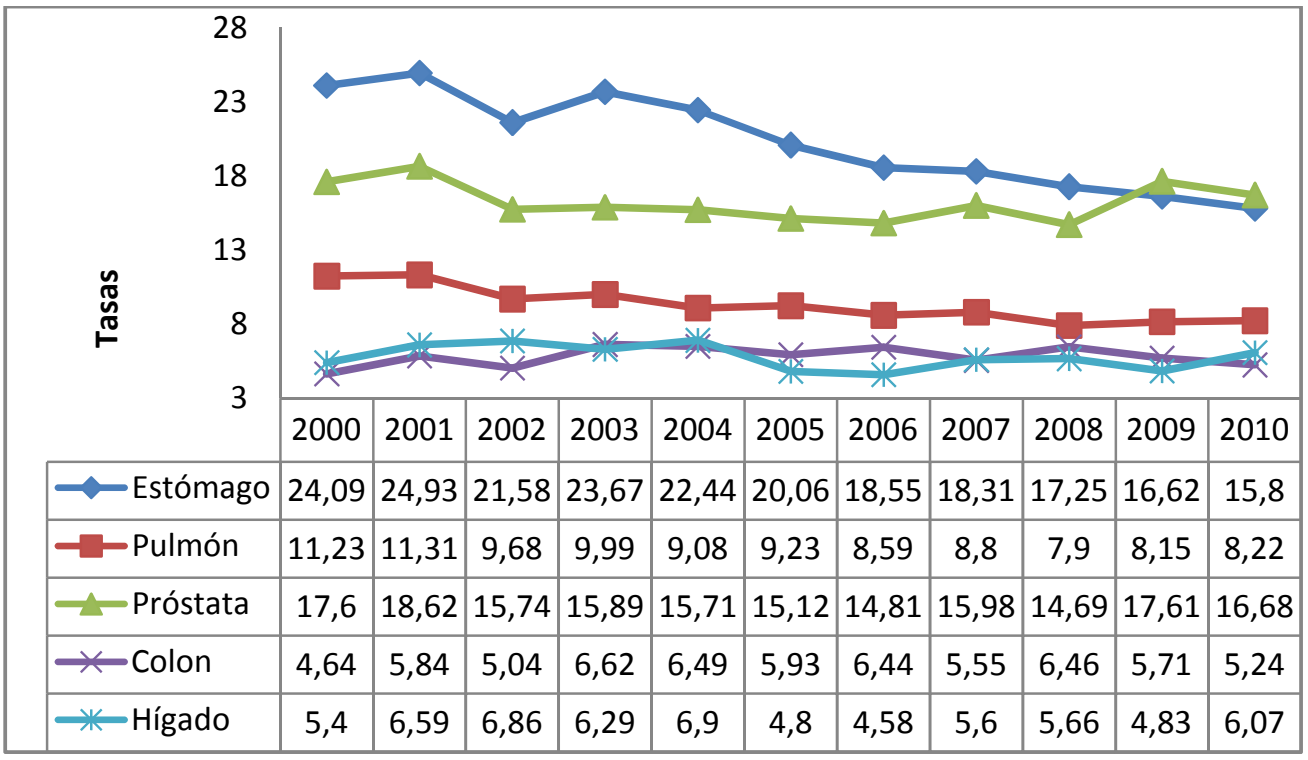

Fuente: INEC - Ministerio de Salud, Dirección Vigilancia de Salud, Unidad Seguimiento de Indicadores de Salud, Costa Rica

Fig. 4: Mortalidad de tumores malignos más frecuentes en mujeres, años 2000-2010. Tasas ajustadas por 100000 mujeres.

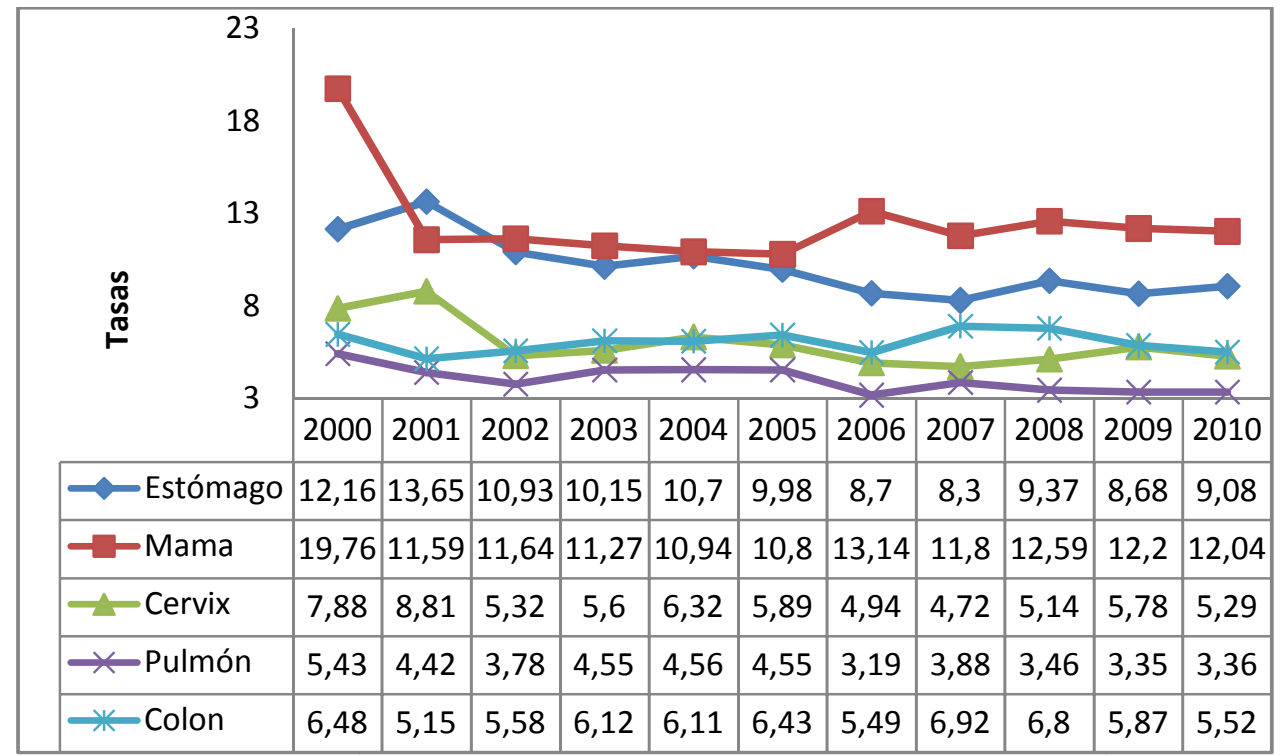

Fuente: INEC - Ministerio de Salud, Dirección Vigilancia de Salud, Registro Nacional de Tumores, Costa Rica 
baja un lugar, ubicándose en el tercero en el el año 2000 pasó al tercero en el 2010, por 2010, solo precedido por el cáncer de estómago encima del cáncer de cérvix (Fig 4 y Fig. 5) [3]. y el de mama; del cuarto lugar que ocupaba en

Fig. 5: Mortalidad por tumores malignos más frecuentes en mujeres año 2010

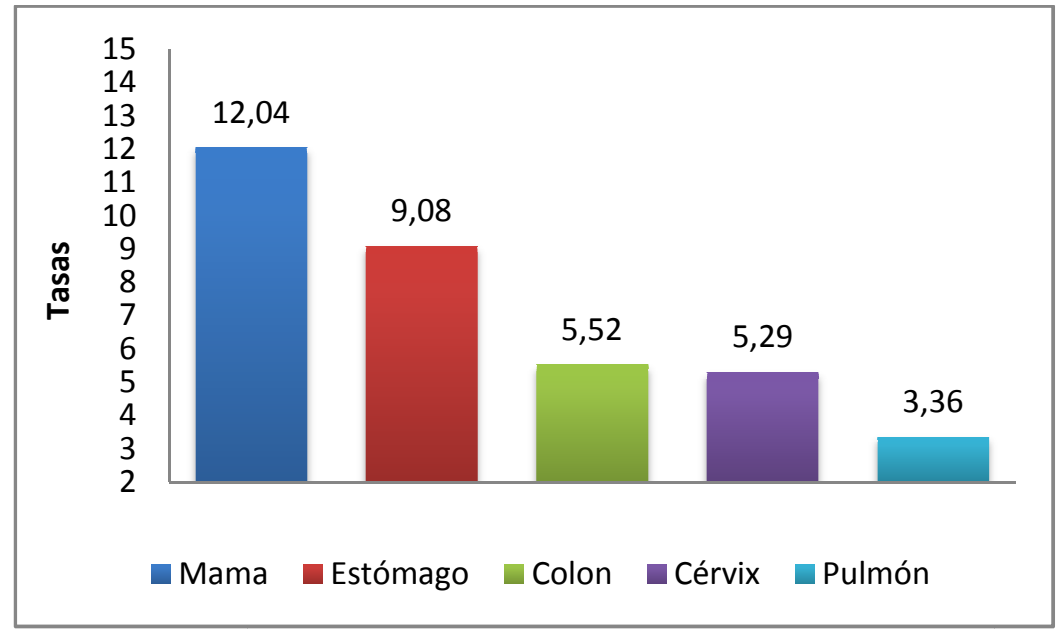

Fuente: INEC - Ministerio de Salud, Dirección Vigilancia de Salud, Registro Nacional de Tumores, Costa Rica

Por mucho tiempo se ha venido postulando la dieta como uno de los factores primordiales en la génesis del cáncer colorrectal, convirtiendo a los países occidentales en la población de más riesgo, debido al alto consumo de productos procesados o con altos contenidos de grasas [4]. Sin embargo, se han realizado seguimientos poblacionales en los cuales personas de países con baja incidencia, migran hacia países de alta incidencia y con esto adoptan los mismos parámetros de riesgo de la población. En algunas partes de Japón, un país altamente industrializado, la incidencia de nuevos casos de cáncer colorrectal ha aumentado hasta en un $90 \%$, debido a la introducción de comidas del hemisferio occidental [5].

\section{FACTORES DE RIESGO MODIFICABLES}

Existe una serie de factores modificables que es necesario conocer con el fin de prevenir una mayor probabilidad de incidencia de este tipo de cáncer. Algunos de estos se detallan a continuación:

\section{Dieta}

La mayor parte de los estudios que han analizado factores dietéticos como protectores $\mathrm{o}$ predisponentes para el cáncer colorrectal ha mostrado resultados contradictorios, por lo que no se pueden extraer recomendaciones firmes con base en la evidencia científica disponible $[4,5,6,7,8,9,10]$; tal es el caso de los suplementos con ácido fólico o magnesio, el consumo de café, ajo o pescados ricos en omega-3. Las dietas ricas en frutas o verduras, así como las ricas en fibra podrían tener un papel protector frente al desarrollo del cáncer de colon, aunque algunos estudios 
han mostrado resultados discordantes [6]. Por el contrario, el consumo elevado de carnes rojas y grasas animales parece aumentar el riesgo de cáncer colorrectal, con base en que la mayor ingesta de carne enlentece el tránsito intestinal y disminuye el lumen del colon, lo que resulta en una prolongada exposición a diversas toxinas. Algunos factores presentan datos más consistentes, como es el caso de la vitamina B6, cuyo efecto en la reducción del cáncer de colon ha sido mostrado en un meta análisis [7].

\section{Fumado}

Después de varios años de debates, un reciente meta análisis que abarcó más de 106 estudios demostró un incremento del $18 \%$ del riesgo entre los pacientes fumadores, principalmente en aquellos con 30 o más años de fumar, con respecto a aquellos que nunca lo habían hecho [8].

\section{Consumo de Alcohol}

En los datos más conservadores, se relaciona la ingesta de 30 gramos de alcohol por día, mientras que en otros menos conservadores se habla de 45 gramos [9]. Sin embargo, la mutagénesis no está bien establecida. Se cree que el acetaldehído como metabolito activo contribuye en la formación de radicales libres, los cuales favorecen el crecimiento de la mucosa colónica; y que a su vez va a favorecer la aparición de pólipos colónicos, los cuales son factores de riesgo para desarrollar cáncer de colon.

\section{Ejercicio y obesidad}

Los beneficios de ejercitarse con regularidad han sido altamente reconocidos para la prevención de este tipo de cáncer, debido a que el ejercicio disminuye los radicales libres y reduce la inflamación del colon, aminorando a su vez las posibilidades de aparición de lesiones en la mucosa colónica.

En un meta análisis de 19 estudios, con hombres y mujeres que se ejercitaban al menos 4 horas por semana, se demostró una reducción del $22 \%$ y el $29 \%$ respectivamente, mientras que la obesidad se asoció al incremento del cáncer colorrectal [11].

\section{FACTORES DE RIESGO NO MODIFICABLES}

Existe una serie de riesgos no modificables que se han venido estudiando y que podrían incrementar la incidencia de este tipo de cáncer.

Raza

Los estudios de registro en los Estados Unidos han demostrado una mayor incidencia de cáncer colorrectal, así como un mayor número de casos por debajo de los 50 años y un predominio por el colon derecho en sociedades orientales [12].

\section{Edad}

En relación con las curvas de incidencia del cáncer de colon en los diferentes grupos de edad, puede afirmarse que el principal factor de riesgo para su aparición es la edad. Así se observa que el $90 \%$ de los casos nuevos de cáncer de colon aparecen por encima de 50 años, siendo muy baja la incidencia en edades menores a los 40 años. La mayor parte de los casos que se desarrolla a edades tempranas suelen corresponder a síndromes hereditarios como se analizará más adelante [1].

Sexo

En estos mismos estudios de registro, se ha podido constatar que los varones presentan un mayor riesgo de desarrollar adenomas colónicos y cáncer colorrectal, así como una mayor mortalidad por este [13].

\section{FACTORES DE RIESGO GENÉTICOS}

Existen múltiples alteraciones genéticas predisponentes para desarrollar cáncer colorrectal. Entre ellas están los síndromes polipósicos como la poliposis adenomatosa familiar, el síndrome de Gardner, el de Turcot y el de Peutz-Jeghers. Además, el síndrome de Lynch tipo I y tipo II, el cual pertenece a la familia de los síndromes no polipósicos [14,15,16,17]. 
La poliposis adenomatosa familiar (PAF) es un síndrome genético de herencia autosómica dominante caracterizado por la aparición de multitud de pólipos adenomatosos (más de 100) desde la infancia. El cáncer se desarrolla antes de los 45 años en más del $90 \%$ de los pacientes no tratados mediante colectomía. Se estima que es el causante del 1\% de los cáncer de colon.

El síndrome de Lynch o cáncer de colon no asociado a poliposis es otra entidad con herencia autosómica dominante, que supone en torno al 3-5\% del total de cáncer de colon. Además de la elevada agregación familiar, destaca la aparición de este tipo de cáncer a edades tempranas, con una edad media de presentación a los 48 años y un predominio por el colon derecho.

Los pacientes con síndrome de Lynch II presentan una mayor incidencia de neoplasias extracolónicas como las de endometrio, estómago, páncreas, biliar, pelvis renal y uréter, vía biliar, ovario e intestino delgado, mientras que en tipo I solo se desarrolla el tumor sin compromiso de ninguna otra estructura.

Otros síndromes hereditarios con riesgo de cáncer de colon más elevado que la población general son el síndrome de Peutz-Jeghers o la poliposis colónica juvenil [14].

\section{ANTECEDENTES FAMILIARES DE CÁNCER DE COLON}

La historia familiar de cáncer de colon, excluidos los síndromes anteriormente descritos, también aumenta el riesgo de padecer este tipo de cáncer en los familiares de primer grado (padres, hermanos e hijos). Esto es de especial relevancia cuando existen dos o más casos de primer grado o ante un único caso, siempre que este fuera diagnosticado antes de los 50-60 años. En el caso de familiares de primer grado con adenomas colónicos de alto riesgo (10 o más mm, displasia de alto grado, componente velloso o múltiples en número igual o mayor que 3 ), existe también un mayor riesgo de desarrollar lesiones de colon [15].

\section{Pólipos \\ Pólipos}

Revista electrónica publicada por el Departamento de Farmacología de la Escuela de Medicina de la Universidad de Costa Rica, 2060 San José, Costa Rica. ${ }^{\circledR}$ All rights reserved. Licensed under a Creative Commons Unported License.

\section{ETIOLOGÍA}

En la actualidad, el conocimiento en la patología molecular del cáncer colorrectal es mayor que en muchas otras neoplasias. La presencia de mutaciones genéticas específicas en línea germinal son las responsables del cáncer de colon que acontece en los síndromes hereditarios como la PAF, mientras que se cree que la adquisición de varias mutaciones somáticas encadenadas sería el origen de la mayor parte de los casos esporádicos. Se sabe que la mayoría de los casos tiene su origen en adenomas colónicos, por lo que la hipótesis de la secuencia adenoma-carcinoma es la más aceptada en la carcinogénesis colónica.

Se han descrito tres mecanismos moleculares diferentes en el proceso de carcinogénesis colorrectal. En primer lugar, se encuentra la inestabilidad cromosómica determinada por mutaciones que 
condicionan un aumento en la expresión de oncogenes o una pérdida de genes supresores de tumores, cuyo ejemplo más característico es el del gen $A P C$, implicado tanto en la PAF como en el cáncer de colon esporádico. El segundo mecanismo se basa en los genes reparadores de alteraciones en el ADN, que condicionan la acumulación de errores genómicos, característicos de los tumores asociados al síndrome de Lynch.

Aproximadamente el $15 \%$ de los casos de cáncer de colon esporádico presenta IMS (Inestabilidad Micro Satélites), debido a la hipermetilación del promotor del gen MSH1, que inhibe su expresión. Este es el tercer mecanismo en la patogénesis del cáncer colorrectal.

\section{MANIFESTACIONES CLÍNICAS}

Los síntomas del cáncer colorrectal tienden a ser inespecíficos. Usualmente se presentan dolores abdominales intermitentes, pérdida de peso, sensación de llenura o sangrado por el recto; en estadios más avanzados de la enfermedad, aparecen náuseas y vómitos.

La presencia de melena se asocia más frecuentemente a cáncer de colon derecho, aunque esto no es exclusivo. Además, se relaciona con la presencia de una masa palpable, y guayacos positivos.

Si el carcinoma se encuentra en el colon izquierdo, se asocia más frecuentemente a dolores tipo cólicos, cambios en el ritmo o patrones intestinales, disminución en el grosor de las heces, hematoquecia o rectorragia.

Una presentación aguda de esta patología también puede ocurrir como una obstrucción intestinal, por lo que si no se le da tratamiento a tiempo, se puede complicar con una isquemia intestinal y posteriormente con una perforación. Sin embargo, pueden aparecer manifestaciones secundarias debido a metástasis; en el caso del cáncer de colon, el principal sitio de metástasis es en el nivel hepático, el cual se da por vía hematógena.

\section{DIAGNÓSTICO}

Siempre se deben tener presentes los factores de riesgo de cáncer colorrectal, para que de esta forma, cuando se revise un paciente, se tenga una perspectiva del tipo de riesgo que se enfrenta, ya sea bajo, moderado o alto.

Un riesgo bajo se presenta en aquella persona de 50 años o mayor, sin antecedentes personales de cáncer colorrectal o de pólipos en el colon, que no tenga familiares en primer grado con historia de pólipos o cáncer colorrectal o dos o menos parientes en segundo grado con historia de este cáncer; este debe de cumplir los cuatro criterios sin excepción.

Uno moderado se hace presente en aquella persona que tenga familiares mayores o menores de 60 años en primer grado con historia de antecedentes de pólipos o cáncer colorrectal, o que tenga dos o más familiares en segundo grado con historia de este cáncer. En este caso con solo una característica de las antes mencionadas se determina riesgo.

Uno alto se presenta cuando existe la presencia de un factor de riesgo genético como el tener un gen para la polipósis adenomatosa familiar o aquellos con cáncer colorrectal hereditario no polipósico [19].

Del grado de riesgo que presente el paciente, dependerán el o los exámenes por realizar. Si el paciente presenta un riesgo bajo para cáncer colorrectal, se deberá hacer, a partir de los 50 años, un examen anual para detectar sangre oculta en las heces. Un examen aun más especializado también puede ser usado anualmente para pacientes con riesgo bajo, el cual consiste en realizar una inmunohistoquímica del material fecal [20]. Posteriormente se cuenta con la sigmoidoscopia flexible, la cual se realiza cada 5 años, al igual que la tomografía axial computarizada y el colon por enema con doble o triple medio de contraste y la colonoscopia que se realiza cada 10 años.

Los pacientes que presenten un nivel de riesgo moderado con historia heredo-familiar de parientes en primer grado con 60 o más años de historia de pólipos o cáncer colorrectal, o con dos o más familiares en segundo grado, deberán iniciar sus respectivos exámenes médicos a partir de los 40 años; estos son los mismos que aquellos que presenten un riesgo bajo. 
Aquellos pacientes con familiares en primer grado, menores de 60 años, con historia de cáncer colorrectal o pólipos, deberán iniciar el screerning a los 40 años o 10 años antes de que el último familiar fuera detectado con la enfermedad.

A los pacientes que sean diagnosticados con poliposis adenomatosa familiar, se debe iniciar su vigilancia a edades tan tempranas como los 10 o 12 años de edad, realizándoles una colonoscopia o sigmoidoscopia anualmente con el fin de determinar extensión y obtener biopsias para estudios posteriores; si estos resultan normales, a partir de los 40 años se puede alargar el tiempo de revisión dependiendo del criterio médico.

Así mismo a los pacientes que presenten cáncer colorrectal hereditario no polipósico, se deberá iniciar su estudio a la edad de 20 a 25 años o 10 años antes de que el último miembro de la familia fuera diagnosticado con la enfermedad. Para este tipo de patología se deberá realizar una colonoscopia cada 6 meses hasta los 40 años y posteriormente de manera anual [21].

Además, antes de la intervención quirúrgica es de mucha importancia realizar una extenuante revisión clínica del paciente para determinar la presencia o no de metástasis. Se pueden usar métodos como la tomografía axial computarizada, el ultrasonido rectal o una resonancia magnética para determinar el estadio del tumor. También son necesarios estudios de laboratorio como hemograma completo, pruebas de función hepática y renal, así como una determinación basal del antígeno carcino embrionario.

\section{TRATAMIENTO}

El tratamiento principal para el cáncer colorrectal es la resección quirúrgica, que dependerá de la localización del tumor primario.

A todos aquellos tumores que se encuentren en el nivel de ciego, flexura hepática o colon ascendente, preferiblemente se les debe realizar una hemicolectomia derecha. A aquellos pacientes con tumores localizados en colon transverso, se les deberá realizar una colectomía transversa. Para los tumores que se encuentren en la flexura esplénica, se puede realizar una resección a partir de la porción media del colon transverso hasta aproximadamente la parte media del colon descendente.

Cuando la tumoración se encuentra en el nivel de colon descendente, se deberá realizar una hemicolectomia izquierda desde la porción media del colon transverso hasta la porción distal del sigmoides. Cuando aparecen tumoraciones rectosigmoidales, confinadas al tercio superior del recto, se deberá realizar una resección anterior y, si el tumor se encuentra en el nivel de tercio medio del recto, se procederá a realizar una resección abdominoperitoneal del tumor, fulguración, resección anterior baja, resección coloanal o terapia radioactiva. Por el contrario, si se presentara en el tercio inferior del recto, se aconseja realizar una resección abdominoperineal, fulguración o terapia radioactiva.

Aquellos pacientes que presenten metástasis hepáticas confinadas al hígado, son candidatos a resección de la tumoración, siempre y cuando no presenten contraindicación intrínseca para la cirugía; a todos los pacientes que tengan evidencia de tumor extra- hepático, o cuya lesión sea muy grande para retirarla, se les ofrece la opción de la quimioterapia, siguiendo los criterios antes mencionados.

Como parte del arsenal terapéutico con el que se cuenta para el manejo del cáncer colorrectal, se ha propuesto el uso de quimioterapia. Según la National Comprehensive Cancer Network, que en un $60 \%$ de los pacientes en estadios III o IV con cáncer colorrectal, la quimioterapia les disminuyó las recidivas y mejoró el pronóstico. Principalmente con el uso de 5- Fluoracilo intravenosa o con capecitabina, que es su equivalente pero en la forma oral. Más recientemente la introducción de otros agentes quimioterapéuticos como el irinotecan y la oxaliplatina han mejorado sustancialmente la sobrevida de aquellos pacientes con cáncer colorrectal [22].

\section{CONCLUSIONES}

El cáncer colorrectal es una patología bien establecida en la sociedad costarricense. Por tal razón, conociendo los factores de riesgo modificables, se podría llegar a bajar la incidencia y mortalidad en la población. 
Es una enfermedad que no tiene que ser sinónimo de muerte inminente, ya que es altamente curable si se descubre en estadios tempranos. Por lo tanto, es indispensable que la población se realice las revisiones médicas respectivas, ya que los principales problemas se presentan cuando la enfermedad está avanzada y muestra metástasis a distancia o se expone de forma aguda con una perforación y sus respectivas complicaciones.

\section{REFERENCIAS}

1. American Cancer Society. Cancer Facts and Figures 2010. Atlanta: American Cancer Society; 2010.

2. Jamal A, Siegel R, Ward E, et al. Cancer statistics, 2007. CA Cancer J Clin 2007;57:43-66.

3. INEC- Ministerio de Salud, Memoria Institucional 2010, Capítulo 4, Dirección Vigilancia de la Salud, Unidad de Seguimiento de Indicadores de salud. Http://www.ministeriodesalud.go.cr/

4. sobre_ministerio/memorias/memoria2011/UMI_ME MORIA_INSTITUCIONAL_2010.pdf

5. Greenfield's surgery, scientific Principles and Practice, Lee J, Demissie K, et al. Cancer incidence among Korean-American immigrants in the United States and native Koreans in South Korea. Cancer Control 2007;14(1):78.85.

6. McMichael AJ ,McCall MG Hartshorne JM, et al. Patters of gastrointestinal cancer in European migrants to Australia: the role of dietary change. Int J Cancer 1980;25(4): 431-437.

7. Negri E, Franceschi S, Parpinel M, La Vecchia C. Fiber intake and risk of colorectal cancer. Cancer Epidemiol Biomarkers Prev. 1998;7:667-71.

8. Larsson SC, Orsini N, Wolk A. Vitamin B6 and risk of colorectal cancer: a meta-analysis of prospective studies. JAMA. 2010;303:1077-83.

9. Nyren O, Bergstrom R, Nystrom L, et al. Smoking and colorectal cancer: a 20 year follow up study of
Swedish construction workers. J Natl Cancer Inst 1996; 88(18):1302-1307.

10. Benedetti A, Parent ME, Siemiatycki J. Lifetime consumption and risk of alcoholic beverages and risk of 13 types of cancer in men: result in a case control study in Montreal. Cancer DetecPrev 2009;32:352362.

11. Kang H, O'Connell JB, Maggard MA, et al. A 10-year outcomes evaluation of mucinous and signet-ring cell carcinoma of the colon and rectum. Dis Colon Rectum 2005;48: 1161-8.

12. Samad AK, Taylor RS, Marshall T, et al. Meta-analysis of the association of physical activity with reduced risk of colorectal cancer. Colorectal Disease 2005;7(3):204-213.

13. Lansdorp-Vogelaar I, van Ballegooijen M, Zauber AG, Boer R, Wilschut J, Winawer SJ, et al. Individualizing colonoscopy screening by sex and race. Gastrointest Endosc. 2009;70:96-108.

14. Edwards BK, Ward E, Kohler BA, Eheman C, Zauber $\mathrm{AG}$, Anderson RN, et al. Annual report to the nation on the status of cancer, 1975-2006, featuring colorectal cancer trends and impact of interventions (risk factors, screening and treatment) to reduce future rates. Cancer. 2010;116:544-73.

15. Jasperson KW, Tuohy TM, Neklason DW, Burt RW. Hereditary and familial colon cancer. Gastroenterology. 2010;138:244-58.

16. Winawer SJ, Zauber AG, Gerdes H, O’Brien MJ, Gottlieb LS, Sternberg SS, et al. Risk of colorectal cancer in the families of patients with adenomatous polyps. National Polyp Study Workgroup. N Engl J Med. 1996;334:82-7.

17. Ahnen DJ. The American College of Gastroenterology Emily Couric Lecture--the adenoma-carcinoma sequence revisited: has the era of genetic tailoring finally arrived? Am J Gastroenterol. 2011;106:190-8.

18. Huang J, Papadopoulos N, McKinley AJ, et al. APC mutations in colorectal tumors with mismatch repair deficiency. Proc Natl Acad Sci U S A 1996;93:904954.

Revista electrónica publicada por el Departamento de Farmacología de la Escuela de Medicina de la Universidad de Costa Rica, 2060 San José, Costa Rica. ${ }^{\circledR}$ All rights reserved. Licensed under a Creative Commons Unported License. 
19. Aldridge AJ, Simpson JN, Histologycal assessment of colorectal adenomas by size, 2001;167(10):777-781.

20. Pignone M, Saha S, Hoerger T, et al,. Cost effectiveness analyses of colorectal cancer screening: a systematic review for the U.S. Preventive Services Task Force. Ann Intern Med 2002; 137(2):96-104.

21. Mandel J S, Church TR, Bond JH, et al. The effect of fecal occult blood screening on the incidence of colorectal cancer. New England Journal Med 2000;343(22)1603-1607.

22. Levin B, Lieberman DA, McFarland B, et al. Screening and surveillance for the early detection of colorectal cancer and adenomatous polyps, 2008:a joint guideline from the American Cancer Society, the US Multi -Society Task Force on Colorectal Cancer and the American College Radiology. Cancer J Clin 2008; 58(3):130-160.

23. Benson AB III, Schrag D, Somerfield MR, et al. American Society of Clinical Oncology recommendations on adjuvant chemotherapy for stage III colon cancer. J Clin Oncol 2004; 22(16): 3408-3419.

24. Macdonald JS. Carcinoembryonic Antigen Screening: pros and cons. Semin Oncol 1999; 26: 556-560.

25. Nelson RL. Postoperative evaluation of patients with colorectal cancer.Semin Oncol 1995; 22: 488-493.

26. Desch CE, Benson AB, Smith TJ, Flynn TJ, Kruse C, Loprinzi CL et al. Colorectal surveillance guidelines by the American Society of Clinical Oncolgy. J Clin Oncol 1999; 17: 1312-1321.

27. Colorectal Cancer Collaborative Group. Adjuvant radiotherapy for rectal cancer: a systematic overview of 8507 patients from 22 randomised trials. Lancet 2001;358(9290):1291-1304.

28. Resection of the liver colorectal carcinoma metastases: a multi- institutional study indications for resection. Registry of Hepatic Metastases. Surgery 1988; 103(3):278-288

29. Bates SE. The used and potential of serum markers: New and old. Drugs 1989; 38: 9-18.
30. Bates SE, Longo DL. Use of serum tumor markers in cancer diagnosis and management. Semin Oncol 1987; 14: 102-138.

31. Bosl GJ. Geller NL, Bajorin D. Serum tumor markers and allocation to good -risk and poor risk clinical trials in patients with germ cell tumors. Cancer 1991; 67: 1299-1304.

32. Panteris V, Haringsma J, Kuipers EJ. Colonoscopy perforation rate, mechanisms and outcome: from diagnostic to therapeutic colonoscopy. Endoscopy. 2009;41:941-51.

33. Sosna J, Sella T, Sy O, Lavin PT, Eliahou R, Fraifeld S, et al. Critical analysis of the performance of doublecontrast barium enema for detecting colorectal polyps $>$ or $=6 \mathrm{~mm}$ in the era of CT colonography. Am J Roentgenol. 2008;190:374-85.

34. Tsai CJ, Lu DK. Small colorectal polyps: histopathology and clinical significance. Am J Gastroenterol 1995;90:988-94.

35. Heald RJ, Bussey HJ. Clinical experiences at St. Mark's Hospital with multiple synchronouscancers of the colon and rectum. Dis Colon Rectum 1975;18:6-10.

36. Cappell MS. From colonic polyps to colon cancer: pathophysiology, clinical presentation, and diagnosis. Clin Lab Med 2005;25:135-77.

37. Bussey HJR. Familial polyposis coli: family studies, histopathology, differential diagnosis and results of treatment. Baltimore (MD): Johns Hopkins University Press; 1975.

38. Stryker SJ, Wolff BG, Culp CE, et al. Natural history of untreated colonic polyps. Gastroenterology1987;93:1009-13.

39. Coebergh JW. Colorectal cancer screening in Europe: firstthings first. Eur J Cancer 2004;40(5):638-42.

40. Baxter NN, Goldwasser MA, Paszat LF, Saskin R, Urbach DR, Rabeneck L. Association of colonoscopy and death from colorectal cancer. Ann Intern Med 2009;150(1):1-8. 
41. Ransohoff DF. How much does colonoscopy reduce coloncancer mortality? Ann Intern Med 2009;150(1):50-2.

42. Levin B, Lieberman DA, McFarland B, Andrews KS, Brooks D, Bond J, et al. Screening and surveillance for the early detection of colorectal cancer and adenomatous polyps, 2008: a joint guideline from the American Cancer Society, the US Multi-Society Task Force

43. Miyaki M, Konishi M, Kikuchi-Yanoshita R, et al. Characteristics of somatic mutation of the adenomatous polyposis coli gene in colorectal tumors. Cancer Res 1994;54:3011-20.

44. Vogelstein B, Fearon ER, Hamilton SR, et al. Genetic alterations during colorectal-tumor development. N Engl J Med 1988;319:525-32

45. Kern SE, Fearon ER, TersmetteKW, et al. Clinical and pathological associations with allelic loss in colorectal carcinoma. JAMA 1989;261:3099-103.

46. Myohanen SK, Baylin SB, Herman JG. Hypermethylation can selectively silence individual p16ink4A alleles in neoplasia. Cancer Res 1998;58:591-3.

47. Burri N, Shaw $\mathrm{P}$, Bouzourene $\mathrm{H}$, et al. Methylation silencing and mutations of the p14ARF and p16NK4A genes in colon cancer. Lab Invest 2001;81:217-29.

48. Shannon BA, Iacopetta BJ. Methylation of the hMLH1, p16, and MDR1 genes in colorectal carcinoma: associations with clinic pathological features. Cancer Lett 2001;167:91-7.

49. Hassan C, Zullo A, Risio M, et al. Histologic risk factors and clinical outcome in colorectal malignant polyp: a pooled-data analysis. Dis Colon Rectum 2005;48:1588-96.

50. Green JB, Timmcke AE, Mitchell WT, et al. Mucinous carcinoma: just another colon cancer? Dis Colon Rectum 1993;36:49-54.

51. Gryfe R. Clinical implications of our advancing knowledge of colorectal cancer genetics: inherited syndromes, prognosis, prevention, screening and therapeutics. Surg Clin North Am 2006;86:787-817.

52. Gatalica Z, Torlakovic E. Pathology of the hereditary colorectal carcinoma. Fam Cancer 2007; [epub June 13].

53. Janier M, Couderic LJ, Morel P, et al. Kaposi's disease in AIDS: 31 cases. Ann Dermatol Venereol 1987;114:185-202 [in French].

54. Cappell MS, Botros N. Predominantly gastrointestinal symptoms and signs in 11 consecutive AIDS patients with gastrointestinal lymphoma: a multicenter, multiyear study of 763 HIV-seropositive patients. Am J Gastroenterol 1994;89:545-9.

55. Church JM. Clinical significance of small colorectal polyps. Dis Colon Rectum 2004;47: 481-5.

56. Cappell MS. The pathophysiology, clinical presentation, and diagnosis of colon cancer and adenomatous polyps. Med Clin North Am 2005;89:142.

57. Jessup JM, McGinnis LS, Steele GD Jr, et al. The National Cancer Data Base: report on colon cancer. Cancer 1996;78:918-26.

58. Cress RD, Morris CR, Wolfe BM. Cancer of the colon and rectum in California: trends in incidence by race/ethnicity, stage, and subsite. Prev Med 2000;31:447-53.

59. Newcomb PA, Norfleet RG, Storer BE, et al. Screening sigmoidoscopy and colorectal cancer mortality. J Natl Cancer Inst 1992;84:1572-5.

60. Sandhu IS, Bhutani MS. Gastrointestinal endoscopic ultrasonography. Med Clin North Am 2002;86:1289317.

61. Fisher ER, Sass R, Palekar A, et al. Dukes' classification revisited: findings from the National Surgical Adjuvant Breast and Bowel Projects (Protocol R-01). Cancer 1989;64: 2354-60.

62. Boland CR, et al. Malignant tumors of the colon. In: Yamada T, Alpers D, Kaplowitz N, editors. Textbook 
of Gastroenterology. 4th edition. Philadelphia:

Lippincott Williams \& Wilkins; 2003. p. 1940-90.

\section{INFORMACION DE AUTOR:}

Young Leitón, Jean Carlo

Universidad de Ciencias Médicas (UCIMED)

E mail: jcyoung99@gmail.com 